\title{
A Weighted Sustainability Index for Selection of Optimal Operating Plans
}

\author{
W.R. Godoy ${ }^{\text {a }}$, A.F. Barton ${ }^{\mathrm{b}}$ and B.J.C. Perera ${ }^{\mathrm{c}}$ and $\underline{\text { Szemis, } \text { J. }^{\text {a }}}$ \\ a Jacobs, Melbourne, Victoria, Australia \\ ${ }^{\mathrm{b}}$ Federation University, Mt Helen Campus, Victoria, Australia \\ ${ }^{\mathrm{c}}$ Victoria University, Footscray, Victoria, Australia \\ Email: walter.godoy@jacobs.com
}

\begin{abstract}
The Wimmera Mallee Pipeline Project (WMPP) provides reticulated water to 36 towns and about 6000 farms across an area of approximately 2 million hectares and forms part of the Wimmera-Mallee Water Supply System (WMWSS). The WMWSS is a multi-reservoir system located in Western Victoria (Australia) which is operated to meet a range of conflicting interests for water using complex operating rules. Since completion in 2010 , the pipeline has vastly improved efficiencies in the supply of water, with water savings being returned to the environment, existing consumptive use and new development. However, one of the major challenges for managers of these water recovery projects is to determine the most effective or optimal operational strategy to meet the needs of all water users.
\end{abstract}

In Victoria, these often conflicting interests to water have traditionally been addressed through a consultative process supported by surface water simulation modelling. Simulation models attempt to represent all the major characteristics of a system and are suited to examine "what if?" scenarios. Whilst such models are highly effective in demonstrating the effect of changes in system operation, the modelling process is limited to finding one solution at a time for a given set of conditions. Optimisation models have also proven to be effective tools but unlike simulation models are characterised by a numeric search technique and are better suited to address "what should be?" questions. In recent times there has been growing interest in linking optimisation techniques with simulation models in order to build on the strengths of both modelling approaches in the search for optimal solutions. The general structure of this combined modelling technique provides for an iterative process; simulation outputs are used to quantify the effect of candidate solutions which are in turn passed to the search engine to find optimal solutions. The process of selecting the most preferred optimal solution brings together two aspects of multi-objective optimisation, namely; (i) the quantitative characteristics of these solutions relative to other solutions; and (ii) the higher level qualitative information in the form of stakeholders' preferences.

The aim of this study is to incorporate stakeholder preferences into a sustainability index which has been previously used to evaluate and compare optimal operating plans for the WMWSS. In that previous study, Godoy et al. (2015) applied a multi-objective optimisation and sustainability assessment approach to an 18objective function multi-objective optimisation problem (MOOP) which represented a range of interests for water. For the present study, the same interests are described in terms of three broad categories i.e. strong environmental preferences, strong social preferences, and strong preferences for the needs of consumptive users. A weighted sustainability index is presented which incorporates these preferences in the original sustainability index formulation. This weighted sustainability index is used to select preferred optimal operating plans previously found by the optimisation-simulation modelling. The results showed that the weighted sustainability index provided a simple means to incorporate stakeholders' preferences into the selection process and inform the decision maker of a stakeholder's uncertainty about their values and priorities for water.

Keywords: Multi-objective optimisation, Sustainability Index, REALM, Wimmera-Mallee Water Supply System 


\section{INTRODUCTION}

Many of the interests for water that exist in water resources systems are conflicting and non-commensurable which can be generally reduced to multi-objective optimisation problems (MOOPs) in which all objectives are considered important. MOOPs consist of a number of objectives subject to a number of inequality and equality constraints as described by Srinivas and Deb (1994):

$\begin{array}{rll}\text { Minimise/Maximise } & f_{i}(x) & i=1,2, \ldots, I \\ \text { Subject to } & g_{j}(x) \leq 0 & j=1,2, \ldots, J \\ & h_{k}(x)=0 & k=1,2, \ldots, K\end{array}$

The parameter $x$ is a $p$ dimensional vector having $p$ design or decision variables. The aim is to find a vector $x$ that satisfies $J$ inequality constraints $g_{j}(x), K$ equality constraints $h_{k}(x)$ and minimises/maximises $I$ objective functions $f_{i}(x)$. Of particular relevance to this thesis, are those problems where three or more objectives are optimised simultaneously; the so called many-objective (or higher order) MOOPs. Solutions to MOOPs are mathematically expressed in terms of superior or non-dominated solutions. This highlights the difficulty with MOOPs in that there is usually no single optimal solution with respect to all objectives, as improving performance for one objective means that the quality of another objective will decrease. Instead there is a set of optimal trade-offs between the conflicting objectives known as the Pareto-optimal solutions or the Pareto front (Deb, 2001). Deb (2001) describes the ideal multi-objective optimisation procedure as one that involves bringing together quantitative and qualitative information as follows:

"Step 1: Find multiple trade-off optimal solutions with a wide range of values for objectives.

Step 2: Choose one of the obtained solutions using higher-level information.” (Deb, 2001)

Present day water planning processes around the world highlight a desire to move towards sustainable water resources systems that have a common view or shared vision for the operation of the system (Loucks and Gladwell 1999). For this to occur the MOOP needs to be formulated in such a way that it guides the search towards optimal solutions that strive to improve the sustainability of the water resources system. Loucks and Gladwell (1999) argued that sustainable development can only succeed with sustainable water resources systems supporting that development.

Godoy et.al. (2015) presented a combined multi-objective optimisation and sustainability assessment approach which was used to formulate and solve a higher order MOOP for the Wimmera-Mallee Water Supply System (WMWSS). The WMWSS is a multi-reservoir system located in Western Victoria (Australia) which is operated to meet a range of conflicting interests to water using complex operating rules. The MOOP was formulated using 18 objective functions, which were based on 18 performance metrics. Building on the generalised procedure for the formulation of MOOPs proposed by Godoy et al. (2011), the study incorporated the proven sustainability performance metrics described by Loucks (1997) (i.e. reliability, resiliency, and vulnerability) to explicitly account for all interests to water in such a way that contributed towards the overall sustainable operation of the WMWSS. The higher order MOOP was solved using an O-S model which found 56 optimal operating plans which were compared to the base case operating plan. The Sustainability Index $(S I)$ values of these optimal operating plans were presented in terms of their normalised rank. These $S I$ exceedance curves were used as a simplified representation of the Pareto front which served as a useful tool to evaluate and compare the sustainability of the optimal operating plans from the perspective of four individual interests to water (i.e. environmental, social, consumptive, and regulated users' interests) and also collectively in terms of the sustainable operation of the WMWSS. The O-S modelling results showed that at least one of the optimal operating plans was more sustainable than the base case operating plan with respect to the various perspectives considered. The study showed that the SI could be used to explicitly account for all major interests to water given its ability to tailor to the individual needs of a range of goals and aspirations.

The aim of this study is to present the second step of the ideal multi-objective optimisation procedure by introducing a modified version of the $S I$ which incorporates stakeholder preferences and value judgements as sourced from available related studies of the WMWSS. This weighted sustainability index is applied to the O$\mathrm{S}$ modelling results from the earlier (first step) work presented in Godoy et al. (2015) to demonstrate the selection of preferred optimal operating plans.

\section{THE STUDY AREA}

Figure 1 is a schematic of the WMWSS showing 6 environmental water demands (EWDs) and 30 consumptive user demands. Other interests in the WMWSS include the provision for recreation amenity and maintenance 
of water quality at certain storages. Three such storages are selected for this study, namely; Lake Lonsdale and Lake Fyans for the provision for recreation amenity (GWMWater, 2012a; 2012b) and Rocklands Reservoir for the maintenance of water quality (GWMWater, 2011). Common to all users of 'regulated' or stored water is the annual and progressive allocation of water that commences in July and ends in June of each year.

Regulated water is used for supply to consumptive users, EWDs, and to provide for recreation amenity at certain storages. There are many possible combinations for harvest and supply of water within the WMWSS. This requires a complex set of rules to move water around the system so that resources are available at the appropriate time and place to meet the needs of all interests to water (Godoy et al., 2011). The system operator, GWMWater, first presented these operating rules in 2011 for the purposes of describing the status quo in operating rules at that time (GWMWater, 2011).

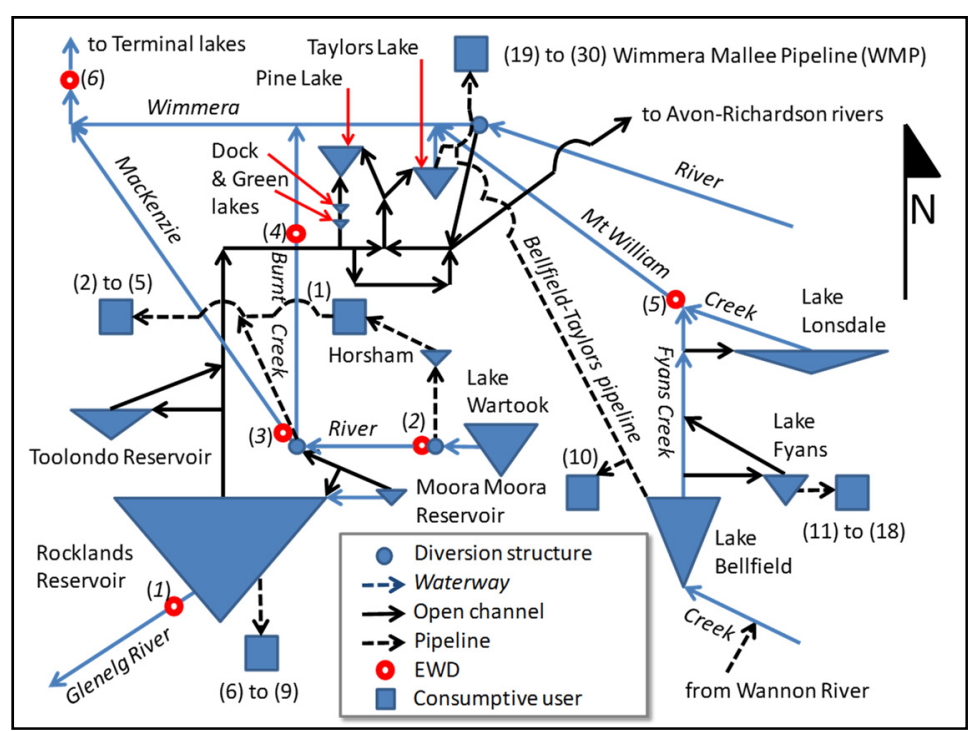

Figure 1. Schematic of WMWSS (not to scale).

\section{SUSTAINABILITY INDEX}

Loucks (1997) developed a sustainability index which can be used to describe the sustainability of water resources systems. This sustainability index combines various performance metrics to represent the reliability, resiliency, and vulnerability of water resources systems over time. Loucks (1997) demonstrated that the sustainability index could be used to evaluate water management policies and to enable the comparison of alternative policies. It is worth highlighting that in an examination of ten performance metrics undertaken by McMahon et al. (2006), the authors considered that the only quantitative measure of system sustainability, which combined reliability, resiliency, and vulnerability, was the sustainability index proposed by Loucks (1997). Loucks and Gladwell (1999) and Sandoval-Solis et al. (2011) further developed the concept of the sustainability index and introduced a multiplicative aggregation scheme to improve its scalability so that it did not obscure poor performance with respect to any one of its performance metrics. One of the major benefits of this sustainability index is that it can be used to summarise the performance of alternative policies from the perspective of different water users.

As explained in Godoy et al. (2015), the Component-level Index $\left(C I_{i}\right)$ assumed that the sustainability for the $i^{\text {th }}$ interest for water was measured in terms of three metrics viz. reliability $\left(\operatorname{Rel}_{i}\right)$, resiliency $\left(\operatorname{Res}_{i}\right)$, and vulnerability $\left(V u l_{i}\right)$. The interests for water identified for the WMWSS were broadly classified into four groups viz. environmental (env); social interests (socio) such as for recreation at Lake Lonsdale $(L L)$, Lake Fyans $(L F)$, and Rocklands Reservoir $(R R)$; consumptive interests (cons); and all these interests collectively in terms of system (sys) water allocations $(a l l o c)$. Note that the social interests were linked to volumes in specific storages whereas for the other groups; the volume held in storage across the whole system (sys) was accounted for by the water allocations (alloc).

Equations (2) to (5) are the Component-level Index for each of these four interest groups as measured by the three abovementioned metrics. Equation (6) is the mathematical expression for the $S I$. The reader is referred to Godoy et al. (2015) for further details regarding the basis of these equations.

$$
\begin{aligned}
& C I_{\text {env }}=\left[\operatorname{Rel}_{\text {env }} \times \operatorname{Res}_{\text {env }} \times\left(1-V u l_{\text {env }}\right)\right]^{1 / 3} \\
& C I_{\text {socio }}=\left[\operatorname{Rel}_{L L} \times \operatorname{Res}_{L L} \times\left(1-V u l_{L L}\right) \times \operatorname{Rel}_{L F} \times \operatorname{Res}_{L F} \times\left(1-V u l_{L F}\right) \times \operatorname{Rel}_{R R} \times \operatorname{Res}_{R R} \times\left(1-V u l_{R R}\right)\right]^{1 / 9} \\
& C I_{\text {cons }}=\left[\operatorname{Rel}_{\text {cons }} \times \operatorname{Res}_{\text {cons }} \times\left(1-V u l_{\text {cons }}\right)\right]^{1 / 3} \\
& C I_{\text {sys }}=\left[\operatorname{Rel}_{\text {alloc }} \times \operatorname{Res}_{\text {alloc }} \times\left(1-V u l_{\text {alloc }}\right)\right]^{1 / 3}
\end{aligned}
$$




$$
S I=\left[\left(C I_{\text {env }}\right)^{3} \times\left(C I_{\text {socio }}\right)^{9} \times\left(C I_{\text {cons }}\right)^{3} \times\left(C I_{\text {sys }}\right)^{3}\right]^{1 / 18}
$$

Godoy et al. (2015) solved the higher order MOOP using an optimisation-simulation (O-S) model which found 56 optimal operating plans. The authors presented the $S I$ values of these optimal operating plans in terms of their normalised rank as shown in Figure 2 and proved that the resulting $S I$ curve was a useful means of evaluating and comparing optimal operating plans in both the objective space and the decision space. Note that this stage represents the use of the $S I$ with respect to the first step of the ideal multi-objective optimisation procedure. Plan no. 46 and Plan no. 11 had the highest $S I$ among the 56 optimal operating plans with values of 0.52 and 0.50 respectively. These two plans are used in Section 5 to demonstrate the selection of a preferred optimal operating plan by incorporating weights into the $S I$ formulation as part of the second step of the ideal multi-objective optimisation

procedure. The (simulation only) base case operating plan (BC01) is also included to show the relative improvement

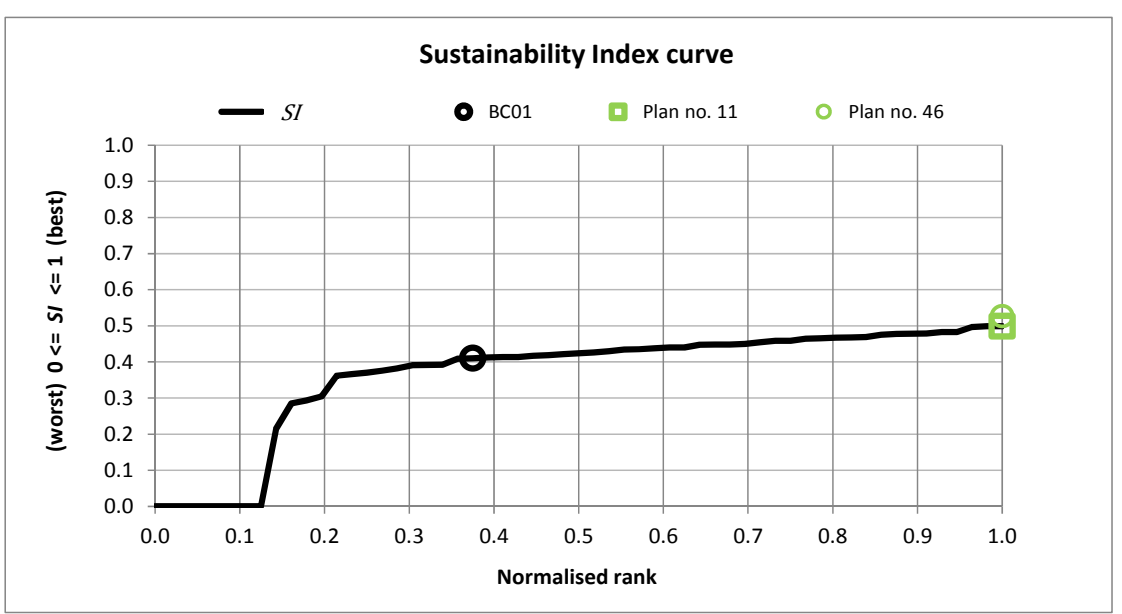

Figure 2. Sustainability Index Curve. in $S I$.

\section{WEIGHTED SUSTAINABILITY INDEX}

The process of selecting a preferred optimal operating plan from the Pareto front brings together two aspects of multi-objective optimisation, namely; (i) the quantitative information regarding the characteristics of the optimal operating plans along the Pareto front; and (ii) the higher level qualitative information in the form of stakeholders' preferences. With reference to the literature on multi-objective optimisation, the quantitative information relating to the optimal operating plans can be analysed in terms of the objective space and the decision space. As in Godoy et al. (2015), the $S I$ was used as the means to evaluate and compare optimal operating plans in both the objective space and the decision space. With respect to the higher level qualitative information, it is necessary to develop a conceptual model which represents stakeholders' preferences and value judgements. Methods available under the umbrella term multi-criteria decision analysis (MCDA) are widely used for the purpose of facilitating the exploration of decisions that take explicit account of multiple factors or criteria (Belton and Stewart, 2002). There are three broad classes of preference models adopted in multi-criteria decision problems viz. value measurement models, goal or reference level models, and outranking models. The use of the $S I$ (in evaluating and comparing optimal operating plans) lends itself to the value measurement preference model. This is due to the $S I$ providing (i) a means of associating a real number for each optimal operating plan; and (ii) an ordering or ranking of these plans, where $S I$ values of 0 and 1 represent the lowest and highest levels of sustainability in the WGWSS respectively. Belton and Stewart (2002) explain that the aforementioned preference models contain two primary components viz. (i) a set of weights which define the relative importance or desirability of achieving different levels of performance for each criterion; and (ii) an aggregation scheme which allows inter-criteria comparisons or trade-offs in order to combine preferences across criteria. The SI provides the basis for these two primary components by allowing for the inclusion of (i) the $j^{\text {th }}$ stakeholder's weight for the $m^{\text {th }}$ performance metric $\left(w_{m}^{j}\right)$; and (ii) a weighted (geometric average) multiplicative aggregation scheme. Thus, the Weighted Sustainability Index $\left(S I^{j}\right)$ for the $j^{\text {th }}$ stakeholder is expressed as follows:

$$
S I^{j}=\left[\prod_{m=1}^{M} P_{m}^{j w_{m}^{j}}\right]^{1 /\left(\sum_{m=1}^{M} w_{m}^{j}\right)}
$$


Where,

$$
\begin{aligned}
& 0 \leq S I^{j} \leq 1 \text { (note: } 0 \text { is the lowest and } 1 \text { is the highest level of sustainability for the } j^{\text {th }} \\
& \text { stakeholder) } \\
& m \text { refers to performance metric, } m=1 \text { to } M \\
& P_{m}^{j} \text { refers to the } j^{\text {th }} \text { stakeholder's } m^{\text {th }} \text { performance metric } \\
& w_{m}^{j} \text { refers to the } j^{\text {th }} \text { stakeholder's weight for the } m^{\text {th }} \text { performance metric }
\end{aligned}
$$

The $S I^{j}$ has all the benefits of the $S I$ in terms of flexibility and scalability and provides continuity in the multicriterial decision-making process i.e. from evaluation and comparison of optimal operating plans through to the selection of a preferred optimal operating plan. Thus, the $j^{\text {th }}$ stakeholder's Weighted Component-level Index $\left(C I_{i}^{j}\right)$ and Weighted Sustainability Index $\left(S I^{j}\right)$ are expressed as follows:

$$
\begin{aligned}
& C I_{e n v}^{j}=\left[\left(\operatorname{Rel}_{e n v}\right)^{w_{1}^{j}} \times\left(\operatorname{Res}_{e n v}\right)^{w_{2}^{j}} \times\left(1-V u l_{e n v}\right)^{w_{3}^{j}}\right]^{1 /\left(\sum_{m=1}^{3} w_{m}^{j}\right)} \\
& C I_{\text {socio }}^{j}=\left[\left(\operatorname{Rel}_{L L}\right)^{w_{4}^{j}} \times\left(\operatorname{Res}_{L L}\right)^{w_{5}^{j}} \times\left(1-V u l_{L L}\right)^{w_{6}^{j}} \times\left(\operatorname{Rel}_{L F}\right)^{w_{7}^{j}} \times\left(\operatorname{Res}_{L F}\right)^{w_{8}^{j}} \times\left(1-V u l_{L F}\right)^{w_{9}^{j}} \times\right. \\
& \left.\left(\operatorname{Rel}_{R R}\right)^{w_{10}^{j}} \times\left(\operatorname{Res}_{R R}\right)^{w_{11}^{j}} \times\left(1-V u l_{R R}\right)^{w_{12}^{j}}\right]^{1 /\left(\sum_{m=4}^{12} w_{m}^{j}\right)} \\
& C I_{\text {cons }}^{j}=\left[\left(\operatorname{Rel}_{\text {cons }}\right)^{w_{13}^{j}} \times\left(\operatorname{Res}_{\text {cons }}\right)^{w_{14}^{j}} \times\left(1-V u l_{\text {cons }}\right)^{w_{15}^{j}}\right]^{1 /\left(\sum_{m=13}^{15} w_{m}^{j}\right)} \\
& C I_{\text {sys }}^{j}=\left[\left(\text { Rel }_{\text {alloc }}\right)^{w_{16}^{j}} \times\left(\operatorname{Res}_{\text {alloc }}\right)^{w_{17}^{j}} \times\left(1-V u l_{\text {alloc }}\right)^{w_{18}^{j}}\right]^{1 /\left(\sum_{m=16}^{18} w_{m}^{j}\right)} \\
& S I^{j}=\left[\left(C I_{\text {env }}^{j}\right)^{1 /\left(\sum_{m=1}^{3} w_{m}^{j}\right)} \times\left(C I_{\text {socio }}^{j}\right)^{1 /\left(\sum_{m=4}^{12} w_{m}^{j}\right)} \times\left(C I_{\text {cons }}^{j}\right)^{1 /\left(\sum_{m=13}^{15} w_{m}^{j}\right)} \times\left(C I_{\text {sys }}^{j}\right)^{1 /\left(\sum_{m=16}^{18} w_{m}^{j}\right)}\right]^{1 /\left(\sum_{m=1}^{18} w_{m}^{j}\right)}
\end{aligned}
$$

Where,

$$
w_{m}^{j} \text {, refers to the } j^{\text {th }} \text { stakeholder's weights for the } m^{\text {th }} \text { performance metric }
$$

Note that the weighted geometric average with equal weights is the same as the geometric average (i.e. $S I^{j}=$ $S I$ and $\left.C I_{i}^{j}=C I_{i}\right)$.

\section{SELECTION OF PREFERRED OPTIMAL OPERATING PLAN}

For the purposes of demonstrating the application of the $S I^{j}$, three sets of preference vectors were gleaned from the available stakeholder information collected as part of recent water resource planning studies of the WGWSS (GWMWater, 2007; 2012a; 2012b; DSE, 2011). These stakeholder preferences are assumed to represent those stakeholders that have (i) higher environmental preferences relating to ecological health of waterways including the flora and fauna that depend on these natural ecosystems $\left(S H^{a}\right)$; (ii) higher social preferences concerning water for recreation and for maintenance of water quality $\left(S H^{b}\right)$; and higher preferences for the needs of consumptive users such as for urban centres, irrigators, and other water-dependent industries $\left(S H^{c}\right)$.

Table 1 summarises the three sets of $C I$ and $S I$ values for two optimal operating plans (i.e. Plan no. 11 and Plan no. 46) as identified by Godoy et al. (2015). The third column provides the aforementioned stakeholder preferences. The fourth and fifth columns present the $C I_{i}$ and $S I$ as calculated by Equations (2) to (6). The sixth and seventh columns present the $C I_{i}^{j}$ and $S I^{j}$ with stakeholder preferences $S H^{a}, S H^{b}$, and $S H^{c}$ applied to them as specified in Equations (8) to (12). By exploiting the aforementioned relationship where the geometric average is the same as the weighted geometric average with equal weights, we can examine the effect of a departure from equal weights with respect to $S I^{j}$. The shaded results in Table 1 represent the best outcome (i.e. the highest values) between corresponding (non-weighted and weighted) $C I$ and $S I$ values. Note that $S H^{c}$ has the same preferences as $S H^{a}$ except for the environmental interests for water and the consumptive interests which are reversed (i.e. $\underline{45}: 22: \underline{6}: 27$ c.f. $\underline{6}: 22: \underline{: 55}: 27)$ ).

With the exception of the consumptive stakeholder preference values for $\boldsymbol{S I}$ and $\boldsymbol{S} \boldsymbol{I}^{\boldsymbol{S}}{ }^{\boldsymbol{c}}$, Table 1 shows that the relativity between Plan no. 11 and Plan no. 46 in terms of any corresponding $\boldsymbol{C I}_{\boldsymbol{i}}$ and $\boldsymbol{C \boldsymbol { I } _ { \boldsymbol { i } } ^ { \boldsymbol { j } }}$ values is the same. In simple terms, environmental and social stakeholders would prefer Plan no. 46 over Plan no. 11 given by the corresponding $\boldsymbol{S I}$ and $\boldsymbol{S I}^{j}$ values. However, the consumptive stakeholder preference values for 
$\boldsymbol{S I}$ and $\boldsymbol{S} \boldsymbol{I}^{S \boldsymbol{H}^{\boldsymbol{c}}}$ show that whilst the $\boldsymbol{C I}_{\boldsymbol{i}}$ and $\boldsymbol{C} \boldsymbol{I}_{\boldsymbol{i}}^{\boldsymbol{j}}$ relativities are the same compared to the corresponding environmental stakeholder preference values, a change in their absolute values can cause a reversal in the relativity of $\boldsymbol{S I}^{\boldsymbol{j}}$ and $\boldsymbol{S} \boldsymbol{I}$ values (i.e. a higher preference for Plan no. 11 over Plan no. 46). This reversal in relativities or turning point, is given by virtue of the multiplicative aggregation approach in the $\boldsymbol{S I}^{j}$. The importance of these turning points with regards to the decision making process is discussed in Section 6. Importantly, the reader is reminded that the $\boldsymbol{S I}^{\boldsymbol{j}}$ should be used as part of the selection process because its formulation incorporates the higher level qualitative information (i.e. weights) which are required to complete the multi-objective optimisation process.

Table 1. Values of Component-level Index and Sustainability Index for two optimal operating plans

\begin{tabular}{|c|c|c|c|c|c|c|}
\hline $\begin{array}{l}\text { Component-level } \\
\text { Index }\left(C I_{i}\right) \text { and } \\
\text { Sustainability } \\
\text { Index }(S I)\end{array}$ & Description & \multicolumn{5}{|c|}{$C I_{i}$ (italic font) and $S I$ (bold italic font) } \\
\hline \multicolumn{2}{|c|}{ Environmental stakeholder preferences: } & $S H^{a}$ & \multicolumn{2}{|c|}{ Values without $S H^{a}$} & \multicolumn{2}{|c|}{ Values with $S H^{a}$} \\
\hline$C I_{\text {socio }}$ & Social Component-level Index - Equations (3) \& (9) & 22 & 0.43 & 0.48 & 0.50 & 0.55 \\
\hline$C I_{\text {cons }}$ & Consumptive Component-level Index - Equations (4) \& (10) & 6 & 0.70 & 0.54 & 0.70 & 0.54 \\
\hline$C I_{\text {sys }}$ & System-wide Component-level Index - Equations (5) \& (11) & 27 & 0.52 & 0.53 & 0.52 & 0.53 \\
\hline$S I$ & Sustainability Index - Equations (6) \& (12) & na & 0.50 & 0.52 & 0.50 & 0.56 \\
\hline \multicolumn{2}{|c|}{ Social stakeholder preferences: } & $S H^{b}$ & \multicolumn{2}{|c|}{ Values without $S H^{b}$} & \multicolumn{2}{|c|}{ Values with $S H^{b}$} \\
\hline$C I_{\text {sys }}$ & System-wide Component-level Index - Equations (5) \& (11) & 22 & 0.52 & 0.53 & 0.53 & 0.54 \\
\hline SI & Sustainability Index - Equations (6) \& (12) & $n a$ & 0.50 & 0.52 & 0.47 & 0.50 \\
\hline \multicolumn{2}{|c|}{ Consumptive stakeholder preferences: } & $S H^{c}$ & \multicolumn{2}{|c|}{ Values without $S H^{c}$} & \multicolumn{2}{|c|}{ Values with $S H^{c}$} \\
\hline$C I_{e n v}$ & Environmental Component-level Index - Equations (2) \& (8) & 6 & 0.52 & 0.61 & 0.52 & 0.61 \\
\hline$C I_{\text {socio }}$ & Social Component-level Index - Equations (3) \& (9) & 22 & 0.43 & 0.48 & 0.50 & 0.55 \\
\hline$C I_{\text {cons }}$ & Consumptive Component-level Index - Equations (4) \& (10) & 45 & 0.70 & 0.54 & 0.67 & 0.49 \\
\hline$C I_{\text {sys }}$ & System-wide Component-level Index - Equations (5) \& (11) & 27 & 0.52 & 0.53 & 0.52 & 0.53 \\
\hline SI & Sustainability Index - Equations (6) \& (12) & na & 0.50 & 0.52 & 0.58 & 0.52 \\
\hline
\end{tabular}

\section{DISCUSSION}

The results of the objective space analysis showed how the $S I^{j}$ could be used to select a preferred optimal operating plan by incorporating stakeholder preferences in the SI. However Belton and Stewart (2002) point out that the determination of an overall value (in a value measurement preference model) should not be viewed as the end of the analysis. The authors explain that the value (i.e. the $S I^{j}$ in this case) ought to be considered as another step in furthering the understanding and promoting discussion about the problem. Indeed, the $S I^{j}$ like the $S I$ is by definition an indicator of the level of sustainability that can be achieved in the WGWSS under a given optimal operating plan. The decision maker needs to be aware that further exploration is required in terms of the composition of the optimal operating plan (i.e. decision space) and in terms of the performance of the WGWSS beyond that provided by the performance metrics alone (i.e. simulation modelling). Similarly, exploration of alternative perspectives of the problem can be undertaken in terms of a sensitivity analysis in order to explore (among other areas) the effect of the stakeholder's uncertainty about their values and priorities or simply to offer a different perspective on the problem.

Belton and Stewart (2002) view this sensitivity analysis from a technical, individual, and a group perspective. The author's describe the technical sensitivity analysis as one that examines the effect of changes to the input parameters of the model on the output of a model. In this way, the analysis sets out to determine the level of influence that the various input parameters have on the overall evaluation (i.e. the $S I^{j}$ in this case). The individual's perspective is to provide a sounding board against which a stakeholder can test their intuition and understanding of the problem. The group perspective often involves the exploration of alternative perspectives, which Belton and Stewart (2002) explain, is often undertaken by using different sets of criteria weights. By using different sets of criteria weights, the turning point(s) at which stakeholders' preference for one optimal solution changes to another optimal solution along the Pareto front can be identified. This informs the decision maker of the effort that ought to be placed on clarifying or confirming a stakeholder's uncertainty about their 
values and priorities. For instance, a higher level of effort would be placed on ascertaining stakeholders' preferences between optimal solutions where stakeholders were highly indecisive about those solutions, compared to solutions in which stakeholders were indifferent.

\section{CONCLUSION}

This study presented the second step of the ideal multi-objective optimisation procedure by introducing a modified version of the Sustainability Index $(S I)$ which incorporates stakeholder preferences and value judgements. This weighted sustainability index $\left(S I^{j}\right)$ was applied to the O-S modelling results from an earlier (first step) work presented in Godoy et al. (2015) to demonstrate the selection of preferred optimal operating plans.

For the purposes of demonstrating the application of the $S I^{j}$, three sets of preference vectors were gleaned from the available stakeholder information collected as part of recent water resource planning studies. These stakeholder preferences were assumed to represent those stakeholders that had (i) higher environmental preferences relating to ecological health of waterways including the flora and fauna that depend on these natural ecosystems; (ii) higher social preferences concerning water for recreation and for maintenance of water quality; and higher preferences for the needs of consumptive users such as for urban centres, irrigators, and other waterdependent industries. The $S I^{j}$ was used to select the preferred optimal operating plan between two plans which were previously found by the optimisation-simulation modelling presented in Godoy et al. (2015). Moreover, this work showed that the weighted sustainability index provided a simple means to incorporate stakeholders' preferences into the selection process and to inform the decision maker of a stakeholder's uncertainty about their values and priorities for water.

\section{ACKNOWLEDGMENTS}

The work presented in this paper was funded by the Australian Research Council through its Project, LP100100554. A special thanks to the reviewers for their important contributions.

\section{REFERENCES}

Belton, V. and Stewart, T.J. (2002). Multiple Criteria Decision Analysis. Kluwer Academic Publishers, United Kingdom.

Deb, K. (2001). Multi-Objective Optimization using Evolutionary Algorithms. John Wiley \& Sons, United Kingdom.

Department of Sustainability and Environment (2011) Western Region Sustainable Water Strategy for Community Comment. Victorian Government, Melbourne, Australia.

Godoy, W. and Barton, A.F. (2011). Modelling the environmental water reserve: A case study exploring the effects of the environment's water entitlement in a complex water supply system. Australian Journal of Water Resources, 14(2), 157-167.

Godoy, W., Barton, A.F., and Perera B.J.C. (2011) "A Procedure for Formulation of Multi-Objective Optimisation Problems in Complex Water Resources Systems," MODSIM2011, 19th International Congress on Modelling and Simulation, Perth, Australia, CD.

Godoy, W., Barton, A.F. and Perera, B.J.C. (2015). A Generalised Procedure for Developing Sustainable Operating Plans for Complex Water Resources Systems. Hydrology and Water Resources Symposium, Newcastle, Australia, CD.

GWMWater (2011), Storage Management Rules for the Wimmera-Mallee System Headworks, prepared for Dept of Sustainability and Environment, Melbourne, Australia.

GWMWater (2012a), Lake Fyans Management Plan, Horsham, Australia.

GWMWater (2012b), Lake Lonsdale Management Plan, Horsham, Australia.

GWMWater (2014), Bulk and Environmental Entitlements Operations Review, Horsham, Australia.

Loucks, D.P. (1997), Quantifying trends in system sustainability, Hydrological Sciences-Journal-des Sciences Hydrologiques, vol. 42(4): $513-530$.

Loucks, D.P., and Gladwell, J.S. (1999), Sustainability Criteria for Water Resource Systems, Cambridge University Press, NewYork, United States of America.

McMahon, T.A., Adeloye, A.J., and Zhou, S-L (2006), Understanding performance measures of reservoirs, Journal of Hydrology, 324 (2006), 359-382.

Sandoval-Solis S., McKinney D. C. and Loucks D. P. (2011) "Sustainability Index for Water Resources Planning and Management," Journal of Water Resources Planning and Management, 137(5), 381-390.

Srinivas, N. and Deb, K. (1994). Multiobjective optimization using nondominated sorting in genetic algorithms. Evolutionary Computation, 2(3), 221-248. 